\title{
Etude d'une faunule madicole de Guadeloupe : compléments à la connaissance des Trichoptères (Trichoptera) de l'Ile
}

\section{Botosaneanu ${ }^{1}$}

Mots clés : habitat/faune madicole, eaux courantes, Trichoptera, Guadeloupe, Petites Antilles.

L'étude de la faune madicole est fort peu avancée aux tropiques. En Guadeloupe, l'étude d'un vaste habitat madicole typique dans le massif de la Soufrière a été entreprise. La faune comporte un nombre d'éléments eumadicoles ou au moins madicophiles : Hydracariens, Diptères, et surtout Trichoptères. Parmi les 10 espèces de Trichoptères appartenant à ces catégories, plusieurs sont soit des nouveautés pour la faune de la Guadeloupe, soit représentées par des $\$$ $\$$ pas encore décrites au niveau de l'espèce pour être nommées ; le cas le plus remarquable est celui de la stricte coexistence de deux espèces de Byrsopteryx, dont une décrite comme nouvelle, et qui se distinguent par la coloration des ailes et par la structure des tergites abdominaux, leurs genitalia $\sigma^{\circ}$ semblant être identiques. Les Trichoptères de 4 cours d'eau auparavant non échantillonnés et appartenant à des zones différentes ont été collectés à la lumière UV, ce qui permet certaines comparaisons. La description de deux espèces endémiques pour la Guadeloupe est complétée. 47 espèces de Trichoptères sont actuellement connues de Guadeloupe.

Study of a madicolous fauna from Guadeloupe : complements to the knowledge of the caddisflies (Trichoptera) from the island

Keywords : madicolous habitat/fauna, running water, Trichoptera, Guadeloupe, Lesser Antilles.

Studies of the madicolous (hygropetric s.l.) fauna in the tropics are limited. In Guadeloupe a large typical madicolous habitat in the Massif de la Soufrière was studied. Its fauna comprises a number of eumadicolous, or at least madicophilic, elements : Hydrachnellae, Diptera and especially caddisflies. Several of the 10 caddisfly species belonging to these categories are either new for the fauna of Guadeloupe, or represented by newly described $\$ \%$ of species not identifiable for the time being; the most remarkable case is that of two strictly coexisting species of Byrsopteryx (one described as new), readily distinguished by their wing pattern and abdominal tergites structure, the $\sigma^{\prime \prime}$ genitalia being apparently identical. The caddisflies of 4 water courses belonging to different zones was sampled by UV light, which enables some comparisons. For two endemic species additional information is made available. At present 47 caddisfly species are known from Guadeloupe.

\section{Introduction : l'habitat madicole de la Deuxième Chute du Carbet}

Le terme "fauna hygropetrica" a été utilisé par Thienemann (1925) pour désigner "die Tierwelt dünn überrieselter Felsen". Dans son travail fondamental sur ce milieu de vie, Vaillant (1956) a parlé de milieu et de faune madicole (pétri-bryo- et limimadicole) en démontrant leur grande originalité. Il s'agit de parois rocheuses inclinées ou verticales, supportant une asso-

1. Zoölogisch Museum (Université d'Amsterdam), Plantage Middenlaan 64, 1018 DH Amsterdam, Pays-Bas. ciation végétale stable, et sur lesquelles l'eau (provenant de suintements, d'embruns de chutes d'eau, ou bien d'éclaboussement par des cours d'eau - "marginal splash zones"-) s'écoule en couche fort mince. Le milieu, caractérisé par la constance du débit - tandis que la température de l'eau peut fluctuer largement - permet le développement d'une faune pauvre en espèces mais remarquable par les adaptations - morphologiques, physiologiques, comportementales - des éléments eumadicoles.

Pour les zones tropicales, l'étude des milieux et des faunes madicoles n'a pas été abordée bien que des espèces eumadicoles remarquables - p. ex. Coléoptères ou Trichoptères néotropicaux - aient été décrites. En Guadeloupe, les vrais habitats madicoles sont rares ; 
on rencontre des parois rocheuses humides dans d'assez nombreux endroits de Basse Terre, notamment sur les côtés de petits sauts d'eau sur les rivières de montagne; mais celles-ci sont pratiquement dépourvues de faune et de flore caractéristiques, car la moindre crue provoquerait leur anéantissement. Une des exceptions concerne l'habitat madicole de grande surperficie et de structure complexe au pied de la Deuxième Chute du Carbet, habitat supportant une association animale stable de grand interêt, qui forme l'objet principal de cette étude. Un premier échantillonnage dans cette station a été réalisé en 1992 (Botosaneanu 1994). Un deuxième échantillonnage a été effectué les 23 et 25 mai 2000, en compagnie de M.L. Redaud, mais dans des conditions météorologiques défavorables.

La Deuxième Chute du Carbet $(110 \mathrm{~m}$ de hauteur ; exposition E) est formée par la Rivière du Grand Carbet, sur le territoire du Parc National de la Guadeloupe. A sa base, et des deux côtés, à ca. $580 \mathrm{~m}$ d'altitude, un vaste habitat madicole se développe ; l'eau qui s'écoule généralement en couche de quelques $\mathrm{mm}$ sur les parois rocheuses verticales, provient des embruns et de suintements (petites veines d'eau latérales par rapport à la cascade). Le substrat est varié : roche couverte par un mince bioderme d'algues, zones à végétation supérieure de taille basse mais dense, accumulations de limon dans des anfractuosités et autres parties abritées de la roche. En mai, la température de l'eau était d'environ $22^{\circ} \mathrm{C}$.

Plusieurs Hydracariens et Diptères eumadicoles ont été récoltés. Les Hydracariens (Limnesiidae : Tyrelliinae) sont représentés par une espèce de Tyrrellia Koenike - également collectée sur un habitat madicole dans la vallée de la Rivière Grande Plaine, $50 \mathrm{~m}$ en aval du Saut Acomat -, et par une espèce de Neotyrrellia Lundblad sûrement inédite (selon R. Gerecke et T. Goldschmidt, in litt.). Ils sont généralement enfouis dans la mince pellicule de limon recouvrant certaines parties de la roche, et deviennent visibles seulement quand ils en émergent.

Plusieurs autres espèces, non eumadicoles, ont été observées. L'habitat est parcouru en tous sens par de nombreux exemplaires de l'Hétéroptère Saldula lomata Polhemus, 1985 (Saldidae); cet élément hygrophile à vaste aréal néarctique et néotropical (Polhemus 1985) est probablement le prédateur le plus actif de cet habitat. Le Gastéropode prosobranche Melanoides tuberculata (O.F. Müller, 1774), à très vaste distribution, est une espèce récemment introduite en Guadeloupe, où elle a été signalée pour la première fois en 1974 et 1976 par J.P. Pointier (Starmühlner 1985) ; une importante population s'est développée dans notre gîte madi- cole, où Melanoides doit être considéré comme élément perturbateur.

\section{Les Trichoptères du gîte madicole}

Quelques exemplaires (O) d'Hydroptila cf. antilliarum Flint, 1968 et de Chimarra haesitationis Bots., 1994, ont été capturés : ce sont, de toute évidence, des madicoxènes.

Seulement deux espèces eumadicoles appartenant à d'autres familles que les Hydroptilidae habitent ce gîte : Xiphocentron fuscum Flint, 1968, et Atanatolica dominicana Flint, 1968. Les galeries des larves de Xiphocentron se développent sur la roche suintante dépourvue de végétation supérieure et de nombreux adultes ont été capturés ; mais l'espèce n'est pas restreinte dans sa distribution aux habitats madicoles typiques, des populations considérables se développant en Guadeloupe sur toute roche éclaboussée en bordure des cours d'eau ou dans leur lit. La situation est radicalement différente pour Atanatolica, dont les adultes et les larves à élégants fourreaux coniques de sable sont fréquemment vus dans le gîte madicole étudié, surtout sur la végétation détrempée.

Trois espèces d'Hydroptilides pourraient être considérées comme madicophiles, mais les deux dernières pourraient fort bien être de vrais eumadicoles.

Un seul ơ de Ochrotrichia ponta Flint, 1968, a été capturé.

La $\$$ non décrite d'une autre espèce d'Ochrotrichia a été capturée ( 2 ex.). Son segment abdominal VII en vue ventrale et ses sclérites vaginaux ont été figurés (Figs 3,4); à ceci il faut ajouter que le tergite VIII est fort développé, sclérotisé, avec des apodèmes attachées en position proximo-latérale, tandis que le sternite respectif est absent. La structure du segment VII ressemble à celle figurée par Flint (1968 : fig. 162) pour $O$. spinosissima Flint, 1964, mais les différences entre les deux sont assez nettes; il est possible qu'il s'agisse de la $\$$ non décrite de $O$. brayi Flint, 1968 - espèce connue uniquement de Dominique : dans ce cas, $O$. brayi serait une addition à la faune des Trichoptères de Guadeloupe.

Enfin, 2 à ailes très foncées et hirsutes et à genitalia bien caractéristiques (Figs 5-7) ont été capturées sur la roche suintante; c'est surtout le segment VII qui est remarquable, avec la paire de longs "lobes" capités (extrémités hirsutes) à la partie distale du tergum, et avec des zones distales fort hirsutes (dorsalement et ventralement). Association et identification spécifique sûres ne sont pas possibles ; mais les genitalia de cette O montrent une assez grande ressemblance avec les fi- 

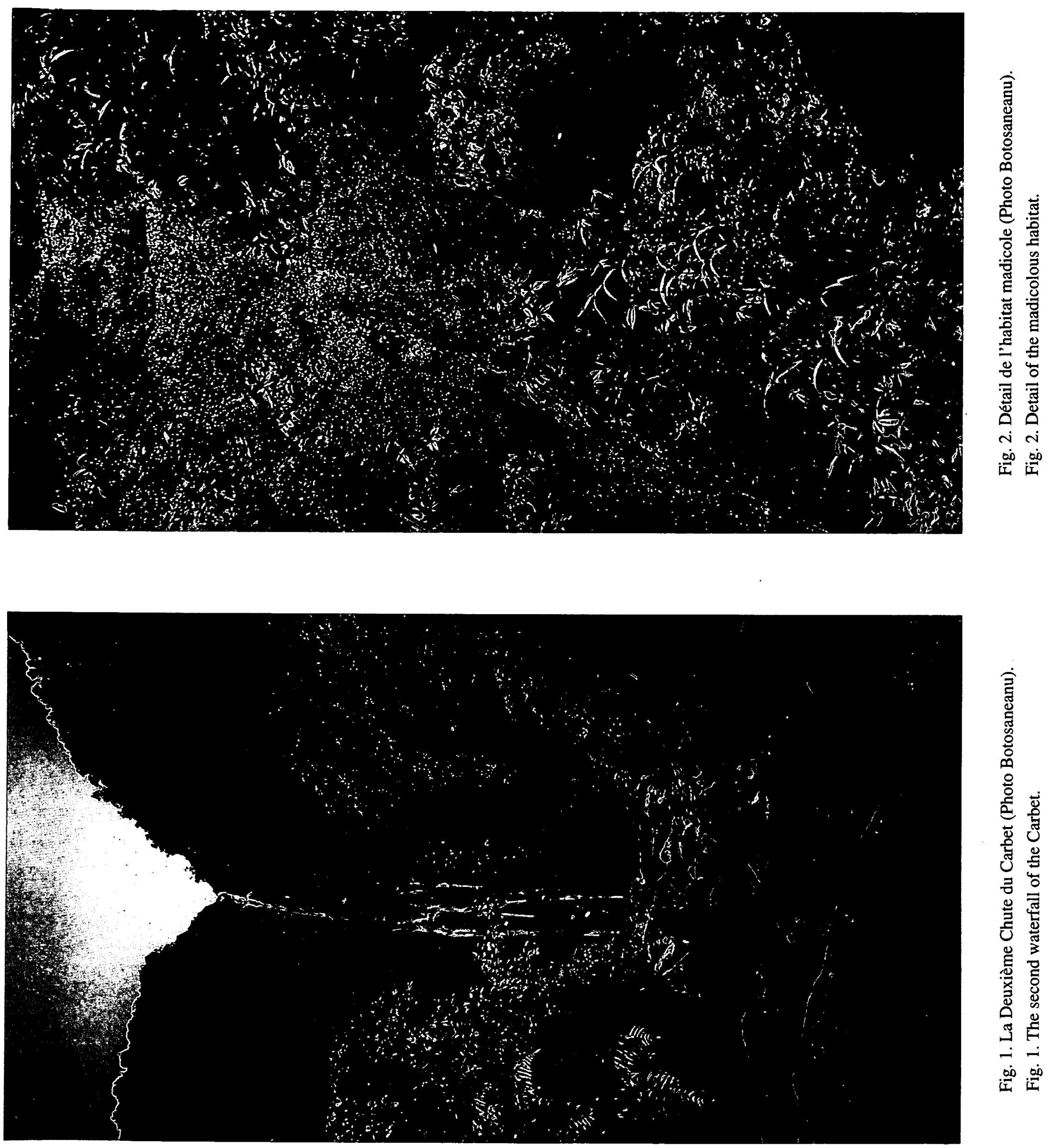
gures publiées (Flint 1968 : Figs. 184-185) pour la $q$ d'une "Ochrotrichia $(M)$ species 2" de Dominica considérée par l'auteur comme étant peut-être celle de Metrichia exclamationis (Flint, 1968) - espèce déjà signalée de Guadeloupe ( $0^{\prime \prime}$; Botosaneanu 1994). Si cette association s'avère être correcte, l'idée formulée par Botosaneanu (1994) que la $q$ à genitalia illustrés fig. 19 pourrait être celle de $M$. exclamationis s'avèrera erronée. L'association correcte des deux sexes des Metrichia des Antilles Françaises reste une tâche d'avenir.

- Metrichia carbetina Bots., 1994, espèce eumadicole, a été retrouvée dans cette localité $\left(10^{\circ}\right.$ ainsi que 2 \% considérées dans Botosaneanu (1994) comme pouvant appartenir à cette espèce).

- Metrichia madicola Bots., 1994, décrite de la même localité, n'a pas été retrouvée.

- Cerasmatrichia wirthi (Flint, 1968), qui est sans doute aussi un eumadicole ( $10^{\prime}$ capturé) est une espèce nouvelle pour la faune de la Guadeloupe. Décrite de Dominique (Flint 1968) elle avait été retrouvée seulement au Venezuela, Edo Aragua et Edo Miranda (Flint 1981) et redécrite par Flint et al. (1994).

Dans Botosaneanu (1994 : 35-36, Figs 5-9) la coexistence sur le gîte madicole de la 2 ème Chute du Carbet de deux espèces distinctes du genre Byrsopteryx Flint, 1981, n'a pu être documentée que sur 2 exemplaires $\%$, aucune identification spécifique n'ayant été possible. Le matériel récolté en 2000 est plus abondant et permet d'éliminer tout doute sur la présence de deux espèces distinctes de Byrsopteryx sur ce site.

$B$. septempunctata a été décrit sur un unique exemplaire, l'holotype ơ de Dominique (Flint 1968) ; une redescription détaillée et bien illustrée de cet exemplaire a été publiée par Harris \& Holzenthal (1994). Une de nos deux espèces est septempunctata - espèce nouvelle pour la Guadeloupe. Dans Botosaneanu (1994) il est fait mention d'une nymphe permettant "de voir vaguement des genitalia mâles rappelant ceux de septempunctata" : cet exemplaire a été réexaminé avec attention : il s'agit effectivement de cette espèce (malheureusement, comme il s'agit d'une nymphe et non d'un métamorphotype, les tergites abdominaux de l'adulte ne sont pas visibles). La chasse de mai 2000 a fourni $15 \%$ de $B$. septempunctata, ce qui me permet de donner une figure détaillée des terminalia en vue ventrale (Fig. 8) ; la partie la plus caractéristique semble être le grand sclérite proximal foncé du segment VIII, pourvu de stries longitudinales et contrastant avec la partie distale pâle du sternum. Les tergites abdominaux de tous ces exemplaires sont parfaitement normaux (non bipartites).
La 2 ème espèce de Byrsopteryx est décrite ci-après comme nouvelle.

\section{Byrsopteryx bipartiterga sp.n.}

Figs 10-13

\section{— Exemplaires examinés}

Holotype $\%, 1$ paratype $\%$, allotype $0^{\circ}, 1$ paratype $\sigma^{\circ}$. Gardés en alcool ou en glycérine au ZMA. Tous ces exemplaires sont des "métamorphotypes" capturés les 23 et 25 mai 2000 : adultes presque parfaitement développés mais n'ayant pas encore quitté les fourreaux nymphaux ; tous permettent une bonne observation des genitalia ; dans le cas de l'holotype $q$, le dessin des ailes antérieures a été bien conservé. Un paratype $\$$ : 10 avril 1992 (ZMA).

$$
\text { tata }
$$

- Description et comparaison avec B. septempunc-

Antennes extrêmement courtes, de 17 articles.

Patron de taches blanches sur l'aile antérieure (Fig. 10). Ce patron, disparaissant aussitôt sur l'aile mouillée, a été bien conservé dans le cas de l'holotype, à l'intérieur du fourreau nymphal étanche. Il est complètement différent de celui de $B$. septempunctata dont un croquis (Fig 8) réalisé sur l'holotype m'a été aimablement fourni par O.S. Flint. Ce patron avait d'ailleurs été décrit par Harris \& Holzenthal (1994) comme étant similaire à celui de leur figure 6 , ce qui correspond presque parfaitement au croquis reproduit ici.

Les tergites abdominaux III-VII des deux sexes sont largement bipartites sur la ligne médiane (Figs 11, 12). Ceci avait été représenté dans Botosaneanu (1994: Fig. 7). O.S. Flint a réexaminé l'holotype de B. septempunctata et m'a confirmé que tous ses tergites abdominaux sont normalement développés.

Genitalia $\$$ (Fig. 13) caractérisés, entre autres, par l'absence du grand sclérite foncé du sternum VIII trouvé chez $B$. septempunctata.

Les genitalia $\sigma^{\circ}$ sont pratiquement identiques à ceux de $B$. septempunctata, parfaitement illustrés par Harris \& Holzenthal (1994 : Fig. 17), ce qui rend, pour l'instant, inutile une illustration pour la nouvelle espèce.

\section{- Observations sur les jeunes stades}

Les nombreuses larves collectées (Fig. 14) sont typiques du genre (Holzenthal \& Harris 1992). Mais il semble que la description du fourreau larvaire donnée (op. cit.) pour B. mirifica Flint, 1981, ne soit pas entièrement valable pour toutes les espèces du genre, car des fourreaux larvaires bien "soudés" sur leurs tranches dorsale et ventrale ont été trouvés. En contraste àvec les larves - toujours trouvées en position latérale dans leurs fourreaux - les nymphes gardent 

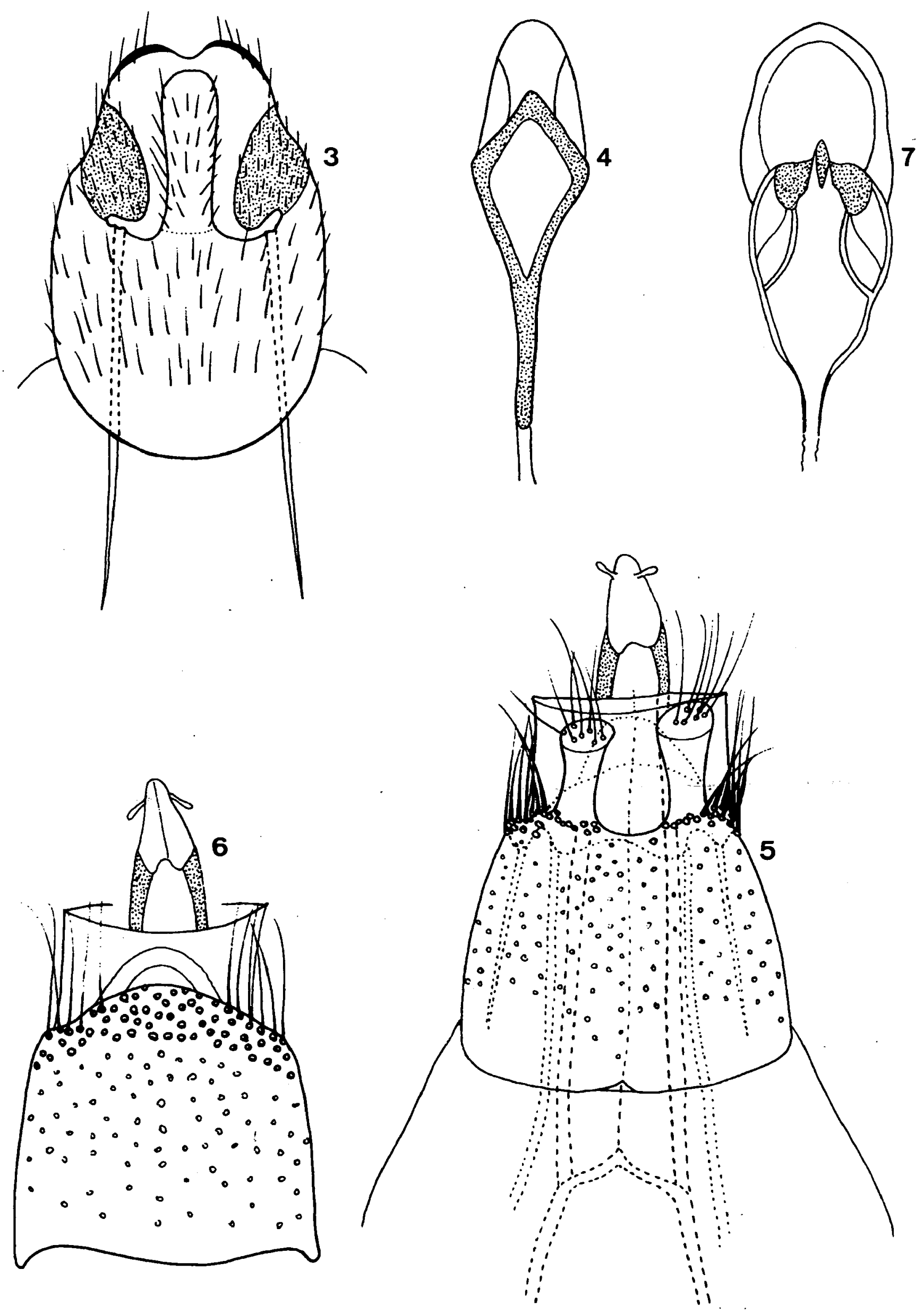

Figs. 3-4. Ochrotrichia cf. brayi 9 , sternum VII (à l'extrémité, le bord du tergum VII est visible), et sclérites vaginaux en vue dorsale. Figs. 5-7. Metrichia sp., $\$$ terminalia en vue dorsale, ventrale, et sclérites vaginaux.

Figs. 3-4. Ochrotrichia cf. brayi $\%$ sternum VII (at apex, the margin of tergum VII is visible) and vaginal sclerites in dorsal view. Figs. 5-7. Metrichia sp., $Q$ terminalia in dorsal view, ventral view, and vaginal sclerites. 

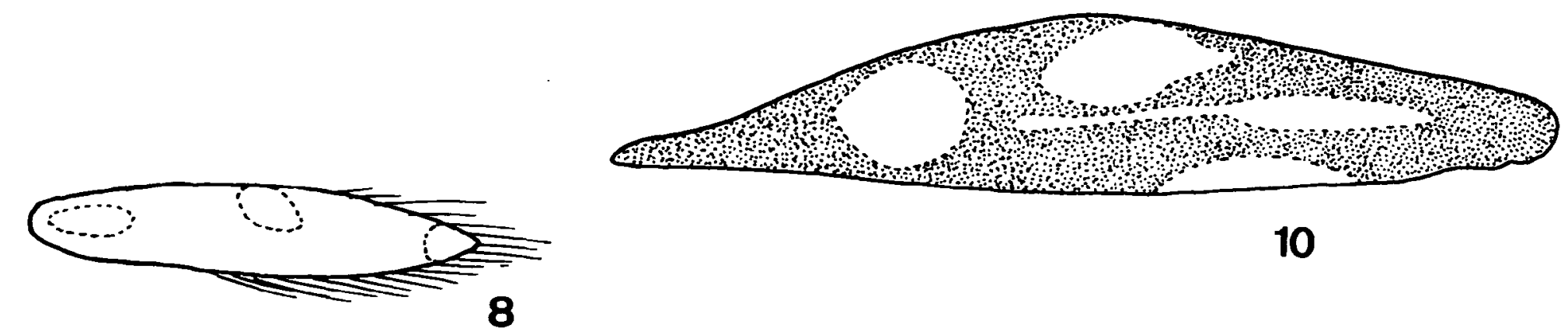

10
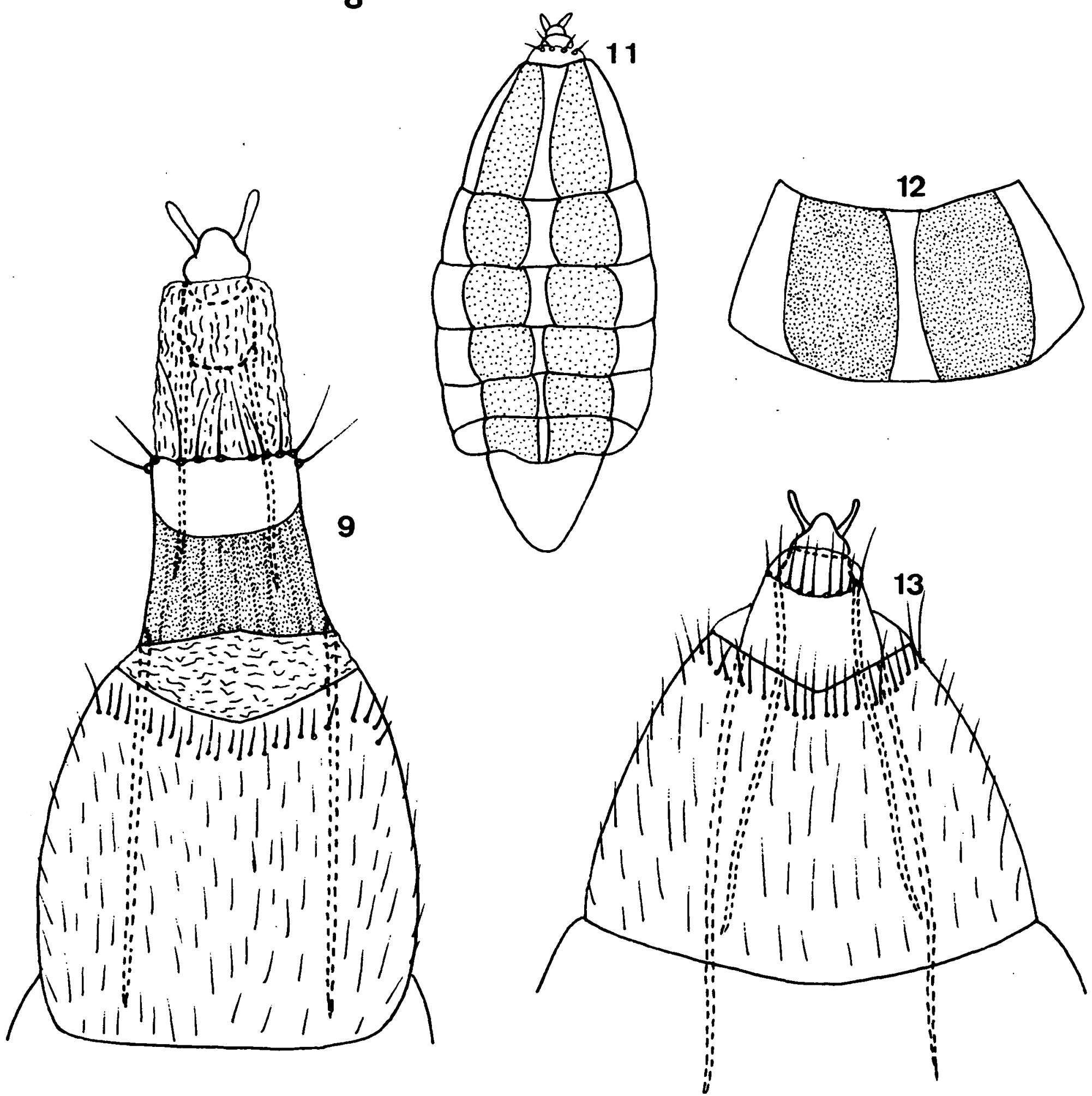

Figs. 8-9. Byrsopteryx septempunctata. croquis de l'aile antérieure de l'holotype $\sigma^{\circ}$ (par O.S. Flint), et terminalia $\$$ - exemplaire de Guadeloupe -, en vue ventrale. Figs. 10-13. Byrsopteryx bipartiterga sp.n., aile gauche de l'holotype $\$$; abdomen en vue dorsale et un des terga abdominaux à plus fort grossissement, paratype ; terminalia de l'holotype $\$$ en vue ventrale.

Figs. 8-9. Byrsopteryx septempunctata, sketch of forewing of the $\sigma^{\circ}$ holotype (by $0 . S$. Flint) and $q$ terminalia - specimen from Guadeloupe - in ventral view. Figs. 10-13. Byrsopteryx bipartiterga sp.n., left wing of female holotype ; abdomen in dorsal view, and one of the abdominal terga of a paratype at higher magnification ; terminalia of the $q$ holotype in ventral view. 

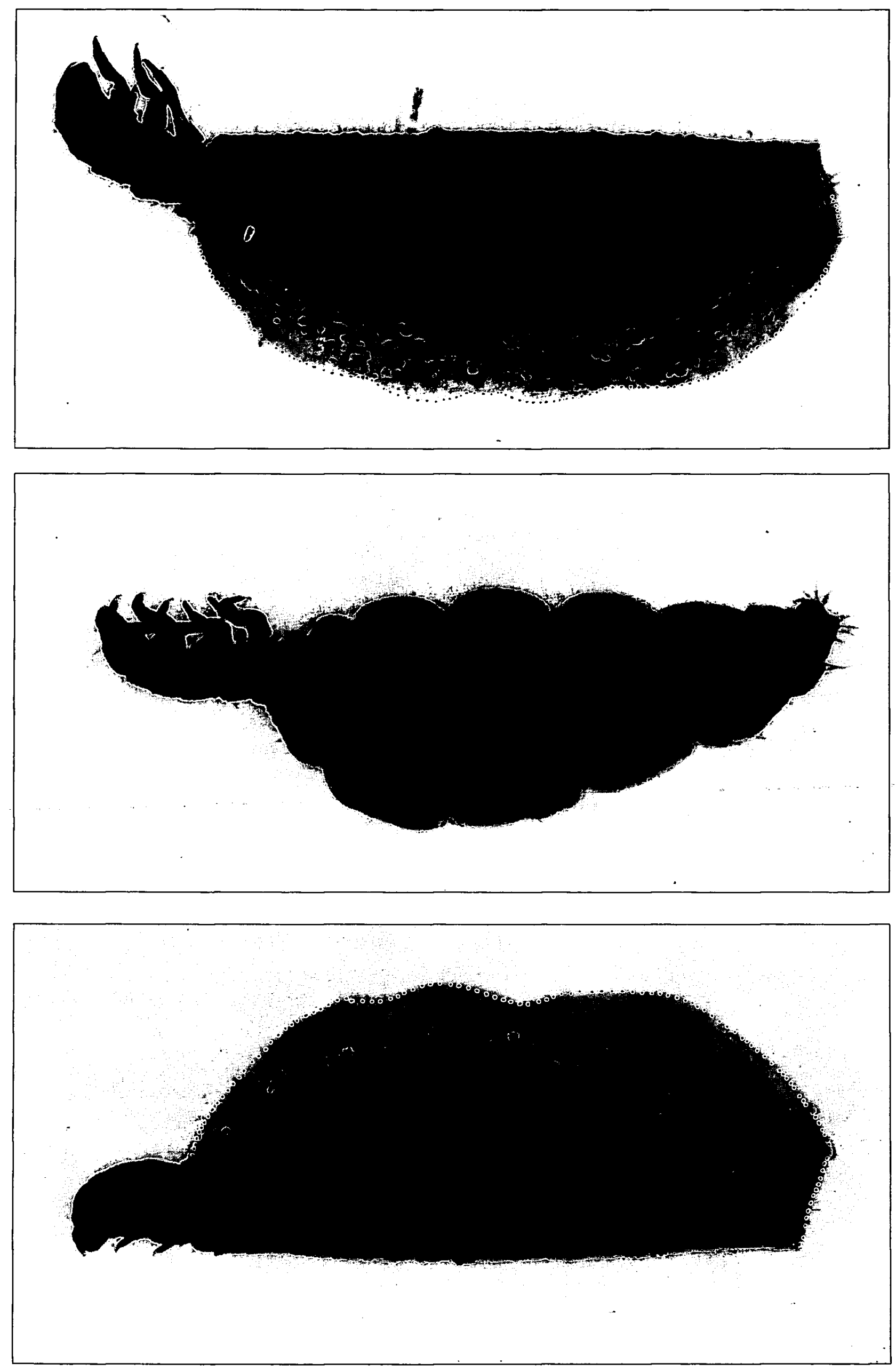

Fig. 14. Byrsopteryx sp., larves (Photos L. van der Laan).

Fig. 14. Byrsopteryx sp., larvae. 
dans leurs fourreaux une position parfaitement dorsoventrale (si la nymphe est observée sur sa face dorsale, le bord dorsal ondulé du fourreau reste à gauche). Le fourreau nymphal est attaché au substrat aux deux extrémités.

\section{- Remarques sur le cas des Byrsopteryx}

La coexistence dans le même habitat madicole de deux espèces étroitement apparentées du genre (genitalia $\sigma^{\top}$ apparemment identiques, différences dans la coloration des ailes, la structure de l'abdomen des deux sexes, les genitalia \%) est un cas intéressant qui mérite une étude plus approfondie. Il est vraisemblable qu'il s'agisse de spéciation sympatrique et de développement d'un "species - specific recognition system" particulier.

Observation généralement valable pour les jeunes stades des Hydroptilides - Byrsopteryx, Ochrotrichia, Metrichia - les larves et nymphes forment surtout des agglomérations mixtes dans de petites anfractuosités de la roche suintante.

\section{La faune de Trichoptères de quatre cours d'eau de Guadeloupe}

Lors de la mission 2000 j'ai pu réaliser des captures de nuit, à la lumière, sur quatre cours d'eau de Basse Terre non échantillonnés lors de la mission 1992. Bien que ces captures (ca. 1300 exemplaires) ne permettent d'ajouter aucune espèce à la liste des Trichoptères de Guadeloupe, elles présentent un certain intérêt, pouvant donner une certaine idée sur l'aspect qualitatif et quantitatif de la faune de cours d'eau de l'hypocrénalépirhitral, du métarhitral, et de l'hyporhitral. D'autre part, elles permettent de donner un complément d'information sur deux espèces connues uniquement de Guadeloupe.

\subsection{Trichoptères de quatre cours d'eau}

1) Petit ruisseau à côté de la Maison du Volcan (La Soufrière). 27.05.2000. L. Botosaneanu \& L. Redaud leg. A $950 \mathrm{~m}$. d'altitude ce ruisselet à eau hypothermale (température d'une source qui s'y jette à l'endroit de la capture : $27,5^{\circ} \mathrm{C}$ ) coule dans un étroit ravin pierreux. Probablement hypocrénal.

Protoptila dominicensis Flint, $1968: 2$ 9 ; Hydroptila martorelli Flint, 1964 : 5 o ; Oxyethira (Loxotrichia) tica Holzenthal \& Harris, 1992 : 5 o", ainsi que 40 \% dont certaines pourraient appartenir à $O .(L$.$) ja-$ nella Denning, 1948.

2) Le ruisseau "Rivière Quatre Bois", affluent de la Rivière Grande Plaine (commune Pointe Noire, quar- tier Marie Jeanne). 26.05.2000. L. Botosaneanu \& Corinne Grac leg. Joli ruisseau typique du métarhitral, à $200 \mathrm{~m}$. d'altitude ; lit pierreux large de 1-2 m. Protoptila dominicensis Flint : 7 0", 59 \& ; Alisotrichia orophila guadeloupea Bots., 1994 : $10^{\circ}$; Zumatrichia antilliensis Flint, 1968: 8 ơ, 5 \% ; Hydroptila antilliarum Flint : 2 \%; Oxyethira (Loxotrichia) tica Holzenthal \& Harris : $1 \sigma^{\circ} ;$ O.(L.) janella Denning : $60^{\circ}$ (et $31 \%$ des deux espèces); Neotrichia iridescens Flint, $1964: 4 \sigma^{\circ}$, 3 ㅇ ; N. tauricornis Malicky. $1980: 4$ o", 8 \% ; N. lacertina Bots., 1994 : 3 o', 2 \% Chimarra (C.) haesitationis Bots. : 4 O', 1 \% ; C. (Otarrha) quadrifurcata Bots., 1994 : 7 o', 3 o ; Smicridea (S.) astarte Malicky, 1980 : 8 ơ, 3 \% ; S. (S.) karukerae Bots., $1994: 1$ \%; Atanatolica dominicana Flint : $20^{\circ}, 1$ \% ; Phylloicus monticolus Flint, $1968 ; 2$ ơ, 1 \& (même observation sur l'apparente variabilité de cette espèce, que dans Botosaneanu, 1994); Helicopsyche guadeloupensis Malicky, $1980: 2$ ơ, 3 \%.

3) Rivière Noire à la Chute du Vauchelet (au dessus du quartier Morne Houel, commune St. Claude). 24.05.2000. Leg. L. Botosaneanu \& Corinne Grac. A ca. $550 \mathrm{~m}$ d'altitude, c'est une assez grande "rivière de montagne" à cours rapide sur lit pierreux contenant aussi de gros blocs rocheux. Hyporhitral.

Protoptila dominicensis Flint : 5 o", 61 \% ; Alisotrichia orophila guadeloupea Bots. : $10^{7}$; Cerasmatrichia dominicensis (Flint 1968) : 2 O', 2 \%; Zumatrichia antilliensis Flint : $290^{\prime \prime}, 40 \%$; Hydroptila martorelli Flint : 8 ơ, 83 \% ; Oxyethira (Loxotrichia) tica Holzenthal \& Harris : 2 ; Chimarra $(C$.) haesitationis Bots. : 36 \% ; . (Otarrha) quadrifurcata Bots. : $30^{\circ}$, 1 \%; Smicridea (S.) astarte Malicky : 8 Ơ, 27 ᄋ ; S. (S.) karukerae Bots. : 4 ơ, 1 \%.

4) Rivière Rouge (commune St. Claude). 23.05.2000. Leg. L. Botosaneanu \& L. Redaud. A ca. $650 \mathrm{~m}$ d'altitude, cette rivière ressemble beaucoup à la précédente.

Protoptila dominicensis Flint : 1 o', 30 \%; Zumatrichia antilliensis Flint : $20^{\circ}, 5 \%$; Hydroptila martorelli Flint : $33 \sigma^{\circ}$, quelques centaines de $\$$; Oxyethira (Loxotrichia) janella Denning et $O$. (L.) tica Holzenthal \& Harris : 110 O", 62 ; Neotrichia lacertina Bots. : $1 \%$; Chimarra (C.) haesitationis Bots. : $6 \%$; C. (Otarrha) quadrifurcata Bots. : $10^{7}$; Smicridea (S.) astarte Malicky : 26 ơ, 77 \& ; Helicopsyche apicauda Flint, $1968: 10$ \%.

Brèves conclusions confirmant ce qui était déjà connu pour les Petites Antilles : il n'y a pas d'individualisation d'une faune des cours d'eau les plus petits - crénal-épirhitral- ; la diversité de la faune atteint un 

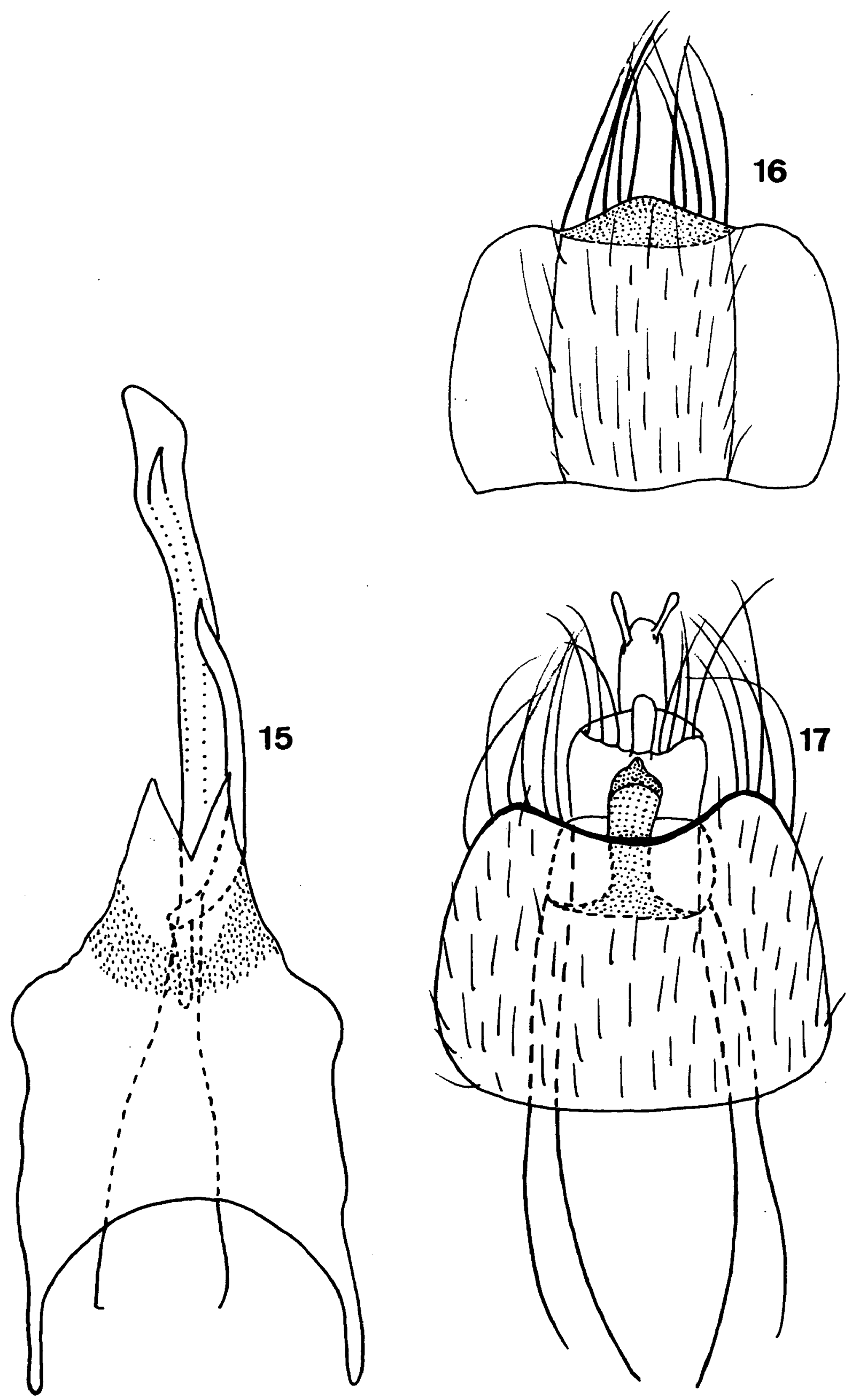

Figs. 15-17. Neotrichia lacertina, genitalia $\sigma^{\circ}$ - segments IX, X et phallus en vue dorsale ; tergum VII de la $\$$; terminalia $\$$ en vue ventrale. Figs. 15-17. Neotrichia lacertina, $\sigma^{\prime}$ genitalia - segments IX, X and phallus, in dorsal view ; $\&$ tergum VII ; $\$$ terminalia, in ventral view. 

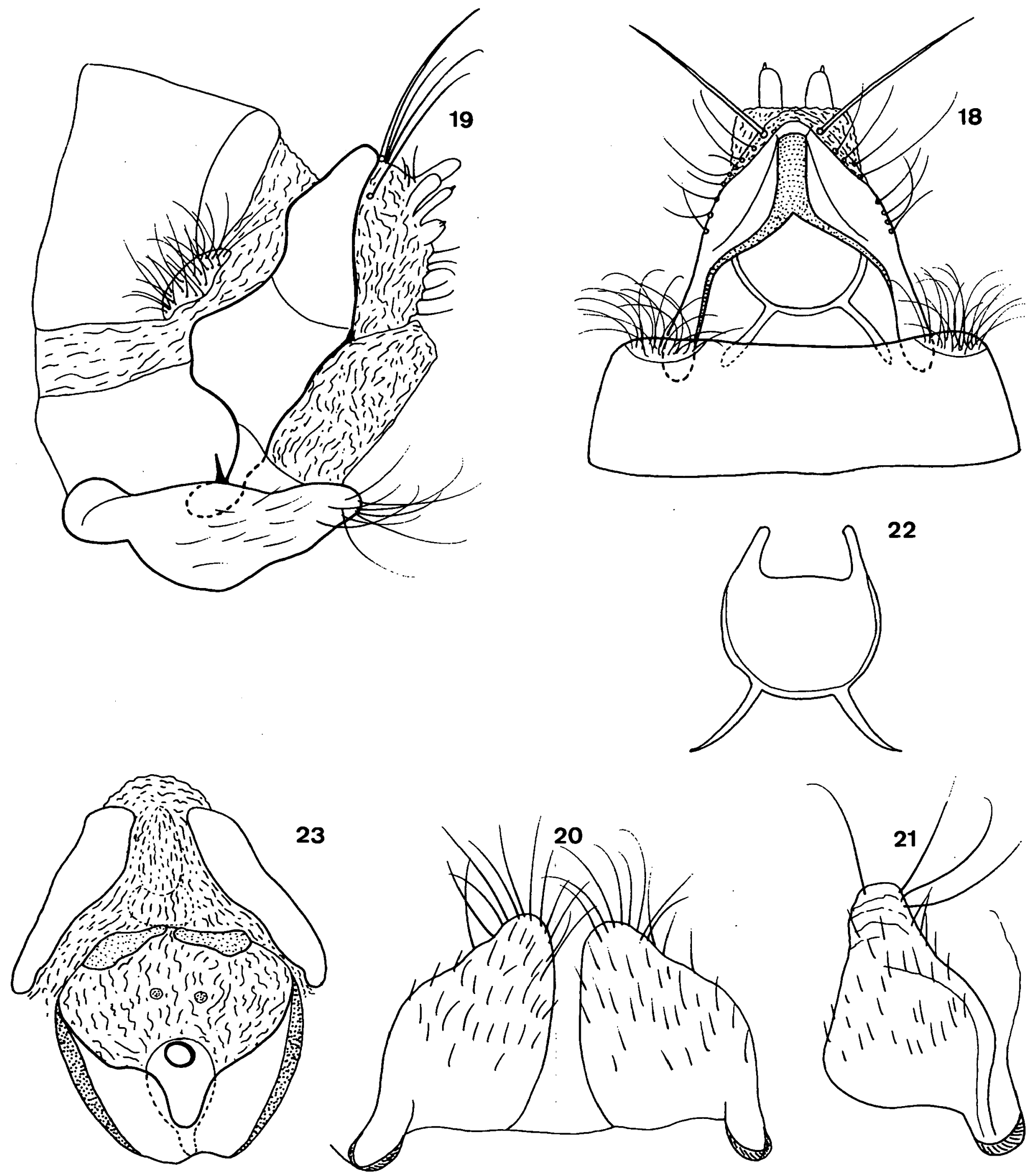

Figs. 18-23. Smicridea (S.) karukerae, genitalia 9 - vue dorsale ; vue latérale ; valves du sternum VIII ; valve gauche du sternum VIII d'un autre exemplaire; "plaque interne" du segment IX ; sclérites vaginaux.

Figs. 18-23. Smicridea $(S)$ karukerae, $\$$ genitalia - dorsal view ; lateral view ; valves of sternum VIII of another specimen ; left valve of sternum VIII ; "internal plate" of segment IX ; vaginal sclerites. 
maximum au niveau des ruisseaux du métarhitral, pour ensuite diminuer légèrement dans les "rivières de montagne" -hyporhitral."

\subsection{Données supplémentaires sur Neotrichia lacerti- na Botosaneanu, 1994}

(Figs 15-17)

\section{- Mâle}

L'apex du segment $X$ est réellement assez profondément fendu ; une partie du segment - mais pas ses pointes apicales - est réellement couverte de minuscules épines. L'extrémité de l'appareil phallique n'a pas été correctement représentée dans la fig. 28 de la description originale : la "forte pointe" n'est en réalité que l'extrémité dévaginable du ductus ejaculatorius, tandis que la "boulette" n'existe pas.

\section{- Femelle}

La femelle de cette espèce restait inconnue. Une particularité assez frappante est le fait que le tibia et le $1^{\text {er }}$ article du tarse des pattes antérieures sont pourvus de nombreuses soies rigides (présentes, mais moins développées, sur le tibia de la $2^{\text {ème }}$ paire de pattes). Tergum VII à tergite bien délimité du reste et se prolongeant vers l'arrière par une projection angulaire pourvue de longues soies ; ce que la fig. 16 ne peut pas montrer est le fait que cette projection est fortement rabattue vers le bas, finissant divisée en deux sclérites triangulaires qui divergent vers l'avant. Sternum VII à bord postérieur profondément émarginé. Segment VIII remarquablement petit, ventralement avec une formation médiane foncée d'aspect caractéristique qui dépasse nettement le bord postérieur du segment et finit en petite pointe émoussée. Sternum IX avec une languette médiane.

\subsection{Données supplémentaires sur Smicridea ( $S$.) $k a$ - rukerae Botosaneanu, 1994}

(Figs 18-23)

Je signale l'existence d'une variabilité intraspécifique qui devrait faire l'objet d'une étude plus poussée : la coloration des ailes antérieures dans différentes populations, parfois brune et parfois noire ; valves du sternum VIII de la $\$$ parfois presque carrées (Fig. 47 : Botosaneanu 1994), parfois à angle médio-distal accusé (Fig. 20) ou fort accusé (Fig. 21).

- Plusieurs détails des genitalia ơ ont été assez médiocrement illustrés ; ci-après nous nous référons aux figures de la description originale : la limite proximale du segment IX en vue latérale (Fig. 42) est plus nettement sinueuse ; $l$ '"entaille" proximale du segment IX en vue dorsale n'est pas toujours comme sur la figure 41, mais plus ouverte antérieurement ; le harpago est plus court que sur la figure 43 ; l'apex de la lèvre ventrale de la phallothèque (Fig. 46) est plus nettement trilobé ; le sclérite médio-dorsal de l'endothèque est plus évasé à l'extrémité (Fig. 45).

- Pour la $q$ je donne une illustration beaucoup plus complète que dans la description originale ; j'attire l'attention sur l'existence de touffes de longues soies aux angles postéro-ventraux du tergum VIII ; la "plaque interne" (segment IX), obliquement placée, a ses "cornes" postérieures plus courtes, dirigées du côté ventral, celles antérieures, plus longues, se dirigeant du côté dorsal ; les sclérites vaginaux sont réprésentés fig. 23.

\section{Remerciements}

Ma mission 2000 à la Guadeloupe a été financée par la Société pour l'Avancement de la Recherche dans les Tropiques (Treub Maatschappij, Amsterdam) et par le Parc National de la Guadeloupe. M. Louis Redaud, à l'époque de ce voyage, chef de la Mission Biodiversité du Parc et Mlle Corinne Grac (Hydrobiologiste, DIREN) ont activement participé au travail sur le terrain. Mes remerciements pour des déterminations s'adressent à Mme le Dr. Pingping Chen (Heteroptera), au Dr. Reinhard Gerecke et à M. Tom Goldschmidt (Hydrachnellae). Je remercie le Dr. O.S. Flint pour des renseignements que je lui avais demandés. Le manuscrit a été saisi par Mme Liesbeth Vermeulen.

\section{Travaux cités}

Botosaneanu L. 1994. - Les trichoptères de la Guadeloupe. Ann. Soc. Entomol. Fr. (N.S.), 30 (1) : 33-54.

Flint O.S. 1968. - The Trichoptera (Caddisflies) of the Lesser Antilles. Proc. U.S.N.M., 125 (no. 3665) : 1-86.

Flint O.S. 1981. - The Trichoptera of the Rio Limón Basin, Venezuela. Smithsonian Contrib. Zool., no. $330: 1-61$.

Flint O.S., Harris S.C. \& Botosaneanu L. 1994. - The description of Cerasmatrichia new genus, a relative of Alisotrichia, with descriptions of new and old species and the larva (Trichoptera : Hydroptilidae). Proc. Biol. Soc. Wash., 107 (2) : 360-382.

Harris S.C. \& Holzenthal R.W. 1994. - Hydroptilidae (Trichoptera) of Costa Rica and the Neotropics : Systematics of the genus Byrsopteryx Flint (Stactobiini). J. New York Entomol. Soc., 102 (2) : 154-192.

Holzenthal R.W. \& Harris S.C. 1992. - The larva of Byrsopteryx mirifica Flint, with an assessment of the phylogenetic placement of the genus within the Leucotrichiini (Trichoptera : Hydroptilidae). Proc. Sixth Internat. Symp. Trich. (C. Tomaszewski ed. ; A. Mickiewicz University Press, Lodz) : 403-407.

Polhemus J.T. 1985. - Shore bugs (Heteroptera, Hemiptera ; Saldidae). A world review and taxonomy of Middle American forms. (The Different Drummer, Eaglewood, Colo., U.S.A.) : 252p.

Thienemann A. 1925. - Die Binnengewässer Mitteleuropas. in Die

- Binnengewässer, 1. E. Schweizerbart'sche Verlagsbuchhandlung, Stuttgart : $255 \mathrm{p}$

Starmühlner F. 1985. - Erstfunde von drei Arten von Süsswasser Gastropoden auf den Inseln Guadeloupe, Dominica und Martinique (Kleine Antillen). Heldia, 1 (2) : 55-58.

Vaillant F. 1956. - Recherches sur la faune madicole (hygropétrique s.l.) de France, de Corse et d'Afrique du Nord. Mém. Mus. Hist. Nat. Paris, $11: 1-258$. 